\title{
Are current scoliosis school screening recommendations evidence- based and up to date? A best evidence synthesis umbrella review
}

\author{
Maciej Płaszewski · Josette Bettany-Saltikov
}

Received: 27 December 2013/Revised: 30 March 2014/ Accepted: 8 April 2014/Published online: 29 April 2014

(C) The Author(s) 2014. This article is published with open access at Springerlink.com

\begin{abstract}
Purpose Recommendations addressing school screening for adolescents with idiopathic scoliosis are contradictory. Consequently a critical evaluation of the methodological quality of available systematic reviews, including those upon which these recommendations are based, was conducted. Methods Articles meeting the minimal criteria to be considered a systematic review were included for a best evidence synthesis, umbrella review of secondary studies. The primary outcome measure was "any recommendation addressing the continuation, or not, of school screening programs". Multiple general bibliographic databases, guideline registries, as well as websites of institutions were searched. The AMSTAR tool was used to critically appraise the methodology of included reviews. Venn diagrams were created to examine potential overlaps across included papers within different reviews.

Results Six reviews undertaken between 2002 and 2011, scored as moderate to low quality, were included. The 2012 US Preventive Services Task Force recommendation against screening was found to be based on an outdated (2004) low-quality review, whilst two higher quality and more recent (2009 and 2010) reviews support the continuation of school screening programs.
\end{abstract}

The online version of this article (doi:10.1007/s00586-014-3307-x) contains supplementary material, which is available to authorized users.

\footnotetext{
M. Płaszewski ( $\square)$

Faculty of Physical Education in Biała Podlaska, Institute of Physiotherapy, Warsaw University School of Physical

Education, ul. Akademicka 2, 21-500 Biała Podlaska, Poland

e-mail: maciej.plaszewski@awf-bp.edu.pl

J. Bettany-Saltikov

School of Health and Social Care, University of Teesside,

Middlesbrough, UK
}

Conclusions As the existing recommendations supporting screening are based on moderate quality evidence whilst the recommendations against screening are based on lowquality evidence, the latter recommendations appear to be both unconvincing and methodologically invalid.

Keywords Idiopathic scoliosis - Screening · Adolescents · Recommendations - Systematic reviews · Quality appraisal

\section{Background}

Adolescent idiopathic scoliosis (AIS) is considered by some authors to be a condition fulfilling the criterion of being an "important healthcare problem meeting the requirements for a screening program" $[1,2]$ : the prevalence is estimated to be at $2-3 \%$ of adolescents aged 10-16 years, the condition, depending on the severity of the deformity and with individual variations, affecting both physical and psychological functioning and with pain and decreased quality of life in the long term [1]. Other authors, however, classify the condition as not meeting this criterion $[3,4]$.

The mandate for school-based screening programs for scoliosis is highly controversial [5-8] and is an issue that is strongly debated [9-14]. National institutions and professional organizations in different countries and/or states currently either opt for mandatory screening [15], recommendation $[2,16-20]$ or simply discourage screening $[1,3$, 6, 21-23]. Even very recent recommendations are contradictory $[2,21,23,24]$. A summary and chronology of the recommendations made by different organizations in different countries since 1979 can be found in Table 1.

Unexpectedly, the 'update' document, based on evidence published prior to 2003 [30], has remained the 
Table 1 Chronology and methods of development of the recommendations addressing school screening for scoliosis

\begin{tabular}{|c|c|c|c|c|}
\hline Year(s) & Institution & Statement & Type & Reference \\
\hline $\begin{array}{l}1970 s- \\
80 s-\end{array}$ & \multicolumn{3}{|c|}{$\begin{array}{l}\text { School screening mandatory/voluntary in the } \\
\text { USA }\end{array}$} & [4] \\
\hline 1979 & CTFPHE & $?$ & EB & [26] \\
\hline $\begin{aligned} 1993 / \\
1996\end{aligned}$ & USPSTF & $?$ & EB & {$[27,28]$} \\
\hline 1994 & CTFPHE & $?$ & EB & [29] \\
\hline 2002 & NHMRC & NO & EB & {$[3]$} \\
\hline 2004 & USPSTF & NO & $\mathrm{EB}$ & {$[30]$} \\
\hline 2005 & Italian guidelines & YES & $\mathrm{CB}$ & [18] \\
\hline 2007 & SOSORT & YES & $\mathrm{CB}$ & [19] \\
\hline 2007 & California $^{a}$ & YES & $\mathrm{EC}$ & {$[15]$} \\
\hline 2008 & $\begin{array}{l}\text { SRS, AAOS, } \\
\text { POSNA, AAP }\end{array}$ & YES & $\mathrm{CB}$ & {$[17,31]$} \\
\hline 2009 & MaHTAS & YES & $\begin{array}{c}\text { EB (SR/ } \\
\text { HTA) }\end{array}$ & {$[16]$} \\
\hline 2010 & & $\begin{array}{l}\text { Meta- } \\
\text { analysis: } \\
\text { YES }\end{array}$ & & {$[32]$} \\
\hline 2011 & AANP & YES & $\mathrm{CPE}$ & {$[33]$} \\
\hline 2011 & AAFP & $\mathrm{NO}^{\mathrm{b}}$ & $\mathrm{EB}$ & {$[23]$} \\
\hline 2011 & ICSI & $\mathrm{NO} / \mathrm{YES}^{\mathrm{c}}$ & $\mathrm{EB} / \mathrm{CB}$ & [34] \\
\hline 2011 & SOSORT & YES & $\mathrm{CB} / \mathrm{EB}^{\mathrm{d}}$ & {$[20]$} \\
\hline 2012 & UK NSC & NO & $\mathrm{EB}$ & [21] \\
\hline 2012 & USPSTF & NO & $\mathrm{EB}^{\mathrm{e}}$ & {$[24,25]$} \\
\hline 2013 & $S R S^{f}$ & YES & $\mathrm{CB} / \mathrm{EB}$ & [2] \\
\hline
\end{tabular}

$N O$ recommendation against, ? unknown effectiveness/insufficient evidence to recommend for or against, $Y E S$ screening recommended, $E B$ evidence-based, $C B$ consensus-based, $O B$ opinion-based, $E C$ educational code, $S R$ systematic review, HTA health technology assessment, CPE continuous professional education paper, CTFPHE Canadian Task Force on Periodic Health Examination; USPSTF US Preventive Services Task Force, NHMRC National Health and Medical Research Council, Australia, SOSORT Society for Spinal Orthopaedic and Rehabilitation Treatment, SRS Scoliosis Research Society, AAOS American Academy of Orthopedic Surgeons, POSNA Pediatric Orthopedic Society of North America, AAP American Academy of Pediatrics, MaHTAS Ministry of Health Malaysia, Health Technology Assessment Section, ICSI Institute for Clinical Systems Improvement, AANP American Academy of Nurse Practitioners, $A A F P$ American Academy of Family Physicians, UK NSC UK National Screening Committee

${ }^{\text {a }}$ Department of Education

b Following USPSTF

c USPSTF and SRS positions considered

d Each recommendation graded according to a "strength of evidence" and/or "strength of recommendation" classification

e Refers to 2004 evidence update

${ }^{f}$ SRS International Panel

background for the 2012 US Preventive Services Task Force (USPSTF) recommendations [25], despite both qualitative [16, 35] and then quantitative [32] systematic reviews favoring screening being published in 2009 and
2010. Conversely, the 2011 review prepared for the United Kingdom National Screening Committee (UK NCS) as an update of the USPSTF 2004 review [30] provides conclusions that discourage screening [1].

Consequently, the recommendations, including those that are evidence-based, remain controversial, with different reviews reaching different conclusions. Hence an analysis of the content and quality of these reviews is urgently needed to help health care professionals as well as service users and commissioners make appropriate policies and evidence-based decisions [36, 37].

Our objective was to critically evaluate the methodological quality of current published systematically developed reviews (including those from systematically developed recommendations), that address school screening for scoliosis.

\section{Methods}

We followed the PRISMA statement for systematic reviews [38] for conducting and reporting this study.

Criteria for inclusion in the review

Types of studies

Eligible studies considered included systematic reviews of any types of primary studies. Papers were considered as systematically developed reviews if they reported on methods to identify and select papers, and which critically appraised relevant evidence [39]. These minimal criteria also applied for reviews of evidence, prepared for, or reported in, systematically developed clinical practice guidelines and recommendations [40]. Exclusion criteria were narrative reviews, expert opinions, letters to the editor and editorials.

The eligibility criteria defined for population, exposure and outcomes are presented in Box 1.

Search strategy

Given the fact that the USPSTF formulated their recommendations based on evidence available between 1994 through 2002, our search was limited, where applicable, to the time period from 01 January 2003 to the most current available date. The databases were last searched between 30 May and 19 August 2013. An updated PubMed and reference list search was also conducted on retrieved papers on 02 November 2013. The search was limited to articles with at a minimum an abstract in English. Databases searched as well as the order of searching are shown in Box 2. 
Box 1 Criteria for inclusion in the review regarding population, exposure/issue and outcomes (PEO)

\begin{tabular}{clc}
\hline & Inclusion criteria & Exclusion criteria \\
\hline Population & $\begin{array}{c}\text { School children, both girls and boys, with no geographical or } \\
\text { other (e.g. societal, racial, cultural) restrictions, in age period } \\
\text { associated with the risk of development of AIS, typically } \\
10-12 \text { years of age; however, no strict age criteria were defined }\end{array}$ & $\begin{array}{c}\text { Papers including other populations of children, e.g. with co- } \\
\text { morbidities, such as Duchenne muscular dystrophy }\end{array}$ \\
$\begin{array}{c}\text { Exposure } \\
\text { or issue }\end{array}$ & $\begin{array}{l}\text { Any reviews addressing 'school screening for AIS' } \\
\text { Outcomes }\end{array}$ & $\begin{array}{c}\text { Papers addressing other screening programs, that did not } \\
\text { exclusively address screening tests for adolescent idiopathic } \\
\text { scoliosis, e.g. general health examinations; screening } \\
\text { programs for other types of scoliosis were also excluded (e.g. } \\
\text { adult scoliosis) }\end{array}$ \\
$\begin{array}{c}\text { Primary outcome: any recommendation that stated to } \\
\text { recommend screening or not to recommend screening, i.e. a } \\
\text { "yes" or "no" with regard to the authors" recommendations; }\end{array}$ & $\begin{array}{c}\text { Not specified } \\
\text { also any secondary outcomes, if analyzed in the retrieved } \\
\text { reviews }\end{array}$ & \\
\hline
\end{tabular}

Box 2 Databases searched and the order of searching

Databases of systematic reviews, guideline registries and databases with separate indexing of systematic reviews and guidelines

The Centre for Reviews and Dissemination databases-DARE, HTA, NHSEED, Cochrane Database of Systematic Reviews (CDSR), Joanna Briggs Institute, Campbell Library, Cochrane Effective Practice and Organisation of Care (EPOC) Group, the AHRQ databases and resource lists from USPSTF, AHRQ Evidence-based Practice Centers (EPC Reports) and National Guideline Clearinghouse, PEDro, INAHTA, TRIP

\section{Websites of institutions}

USPSTF, CTFPHC, NHMRC, UK Screening Portal/ UK NSC Policy Database, Scottish Intercollegiate Guideline Network (SIGN), National Institute for Clinical Excellence, UK (NICE)

\section{General bibliographic databases}

MEDLINE through PubMed, Web of Science and SportsDiscus through EBSCO, Google Scholar

Gray literature-registered protocols, reviews in progress, guidelines in development and registered titles

PROSPERO, CDSR, the USPSTF registry of the topics in progress, the CTFPHC protocols, HSR Project Database, NICE, AHRQ EPC

Reports database (for the EPC Reports in Progress), HSRProj Database, the NHMRC website and the Systematic Review Data Repository (SRDR) database, the Conference Proceedings Citation Index-Science from the Web of Knowledge

For abbreviations not explained here see Table 2

The search terms "scoliosis" and "screening" were used to search the registries of systematic reviews, guideline databases and portals of screening recommendations. In PubMed the MeSH terms "scoliosis" and "mass screening" as well as the free terms "scoliosis" and "screening" were used together with the filters "review" and "meta-analysis". For other bibliographic databases corresponding search terms and limits were used. Additionally, the reference lists of papers included for full text analysis as well as other publications in this area were handsearched. Authors of published papers and reviews in progress were not contacted, as our aim was to assess published reviews.
Study selection

We independently conducted the searches as well as the initial selection of studies by their title and/or abstract. Full papers were then examined for eligibility. Disagreements were resolved by discussion. The PRISMA search flow for the selection of included studies is shown in Fig. 1.

Methodological assessment

The "Assessment of Multiple Systematic Reviews", AMSTAR measurement tool [41] was used to assess the methodological quality of included reviews. The AMSTAR 


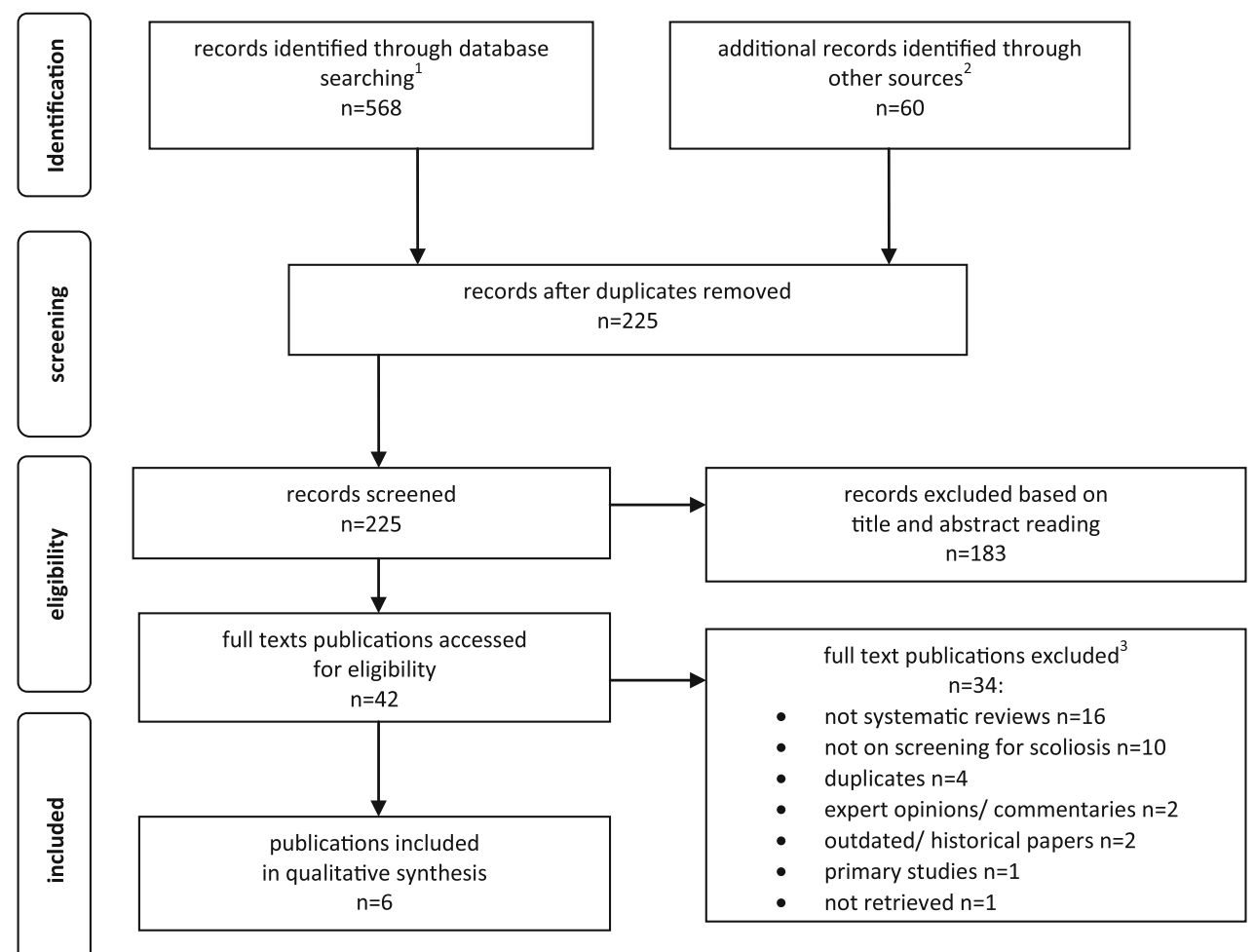

Fig. 1 PRISMA flow diagram of included studies. 1 CRD York (DARE, HTA, NHSEED), $n=72$, Cochrane CDSR, $n=35$, Joanna Briggs Institute, $n=1$, Campbell Library, $n=0$, PEDro, $n=17$, TRIP, $n=14$, PubMed/MEDLINE, $n=109$, Web of Science, $n=203$, SportsDiscus, $n=13$, GoogleScholar, $n=104 ; 2$ registries, websites of institutions: Cochrane EPOC Group, $n=0$,

comprises 11 quality criteria (Table 3). The items are scored "yes", "no", "cannot answer", or "not applicable". The maximum score is 11 . Scores $0-4,5-8$ and $9-11$ indicate low-, moderate- and high-quality reviews, respectively [42]. The appraisal was conducted independently by MP and JB-S, using guides for scoring AMSTAR questions [41, 42]. Disagreements were resolved by discussion and compromise.

Further, the quality analysis of the USPSTF review [30] was expanded with a search for information addressing AMSTAR questions in related USPSTF documents, referenced in the appraised paper [27, 28, 43, 44]. When appraising the UK NSC [1] and NHMRC [3] reviews, the supplementing documentation was also evaluated [45-48].

\section{Overlap across studies}

The reference lists of the included reviews were compared and Venn diagrams were created to examine potential overlaps across included papers within different reviews (Figs. 2, 3, 4, 5, Table A3-supplementary material).
USPSTF, $n=3$, AHRQ EBP Reports, $n=0$, National Guideline Clearinghouse, $n=25$, INAHTA, $n=1$, CTFPHC, $n=1$, SIGN, $n=0$, NICE, $n=15$, UK NSC, $n=5$, NHMRC, $n=4$, HSRProj, $n=2$, PROSPERO, $n=2$; hand searching of reference lists, $n=2$; 3 some full text papers were excluded for more than one reason

\section{Findings}

Search results

From a total of 224 papers, six articles met the criteria for inclusion within the analysis: two quantitative systematic reviews [1,32] one of which included a meta-analysis [32] and four systematic analyses of evidence which were part of $[3,16,18]$ or supplementing [30] recommendation documents. Two further publications were duplicates [35, 49]. One supplement [48] was excluded as it duplicated the content of the resource review [3]. The included papers are characterized in Table 2. Excluded publications are listed, with reasons for exclusion, in Table A2 (supplementary material).

\section{Gray literature}

A protocol [50] as well as an information statement was found [2] together with a reference to one systematic review being in press [51]. However, as the paper was not published at the time of writing this manuscript, it was not 


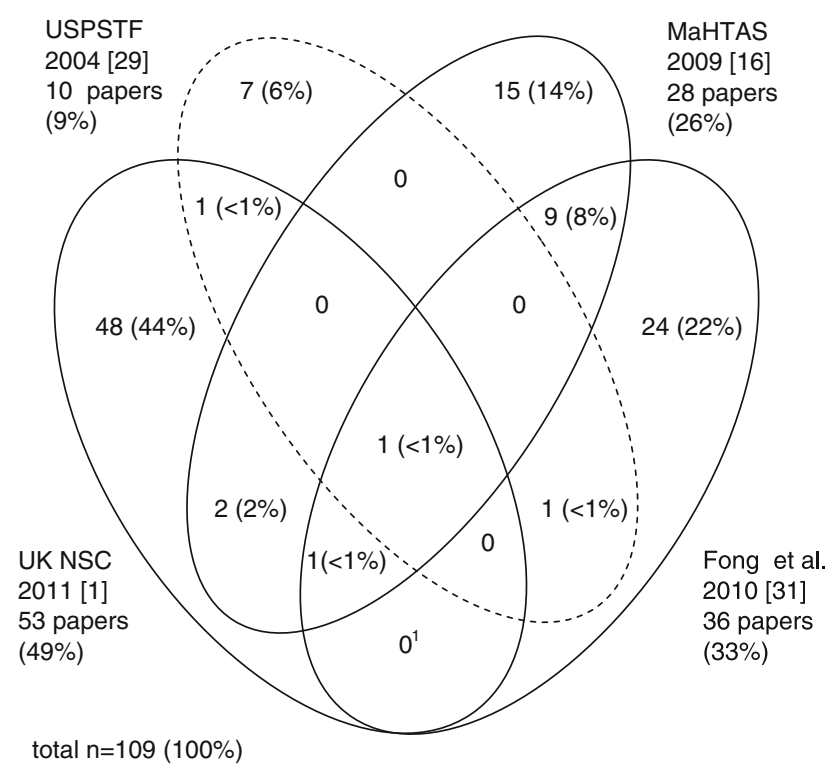

Fig. 2 Venn diagram illustrating the overlap of papers included in the USPSTF report and the more recent systematic reviews. Numbers and percentages inside the ellipses show the overlap of studies included in the four reviews. Percentages outside the ellipses illustrate the percentage of all 109 studies included in each of the four reviews. Total $n=109(100 \%)$. USPSTF-the US Preventive Services Task Force; MaHTAS Health Technology Assessment Section, Ministry of Health Malaysia; UK NSC the UK National Screening Committee; 1 this review also included the systematic review by Fong et al.

included in the analysis. The review is, however, listed in Table A2 (appendices) — the list of excluded papers.

Quality analysis

Three papers $[3,16,32]$ were of moderate and three $[1,18$, 29] of low quality, respectively. The review by Sabirin et al. [16] and the meta-analysis by Fong et al. [32] received the highest scores of 6 points. The NHMRC [3] and the UK NSC [1] reviews received scores of 5 and 4 , respectively. The Italian guidelines [18] as well as the USPSTF review [30] received the lowest scores of 2 points. Table 3 provides details of the AMSTAR quality assessment, with explanations regarding the scoring decisions.

\section{Overlap across included reviews}

Included reviews differed substantially with regard to both the numbers as well as the choice of included papers. The USPSTF evidence analysis [30] included 10 studies (with $14 \%$ of reports included in all reviews), while Sabirin et al. [16] and Fong et al. [32] included 28 and 36 papers (41 and $52 \%$ of reports included in all analyzed reviews), respectively. The USPSTF and the NHMRC recommendations that are based on 10 and 13 papers, respectively, had only three papers overlap (Figs. 2, 3).

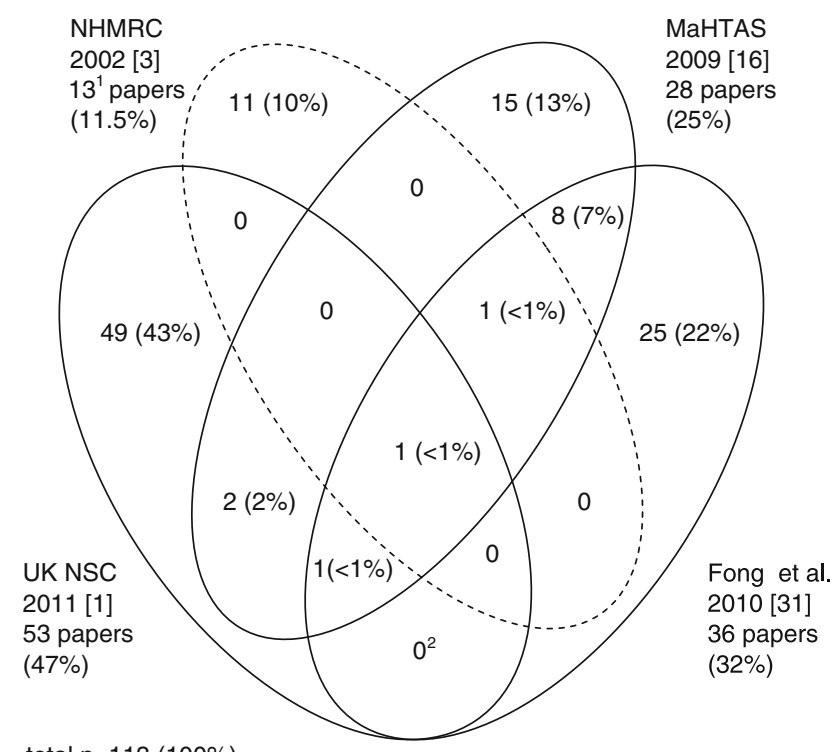

total $n=113(100 \%)$

Fig. 3 Venn diagram illustrating the overlap of papers included in the NHMRC report and the more recent systematic reviews. Numbers and percentages inside the ellipses show the overlap of studies included in the four reviews. Percentages outside the ellipses illustrate the percentage of all 113 studies included in each of the four reviews. Total $n=113(100 \%)$. NHMRC National Health and Medical Research Council, Australia; MaHTAS Health Technology Assessment Section, Ministry of Health Malaysia; UK NSC the UK National Screening Committee; 1 quality assessment for four key articles documented in the report; 2 Fong et al. is included in the UK NSC review

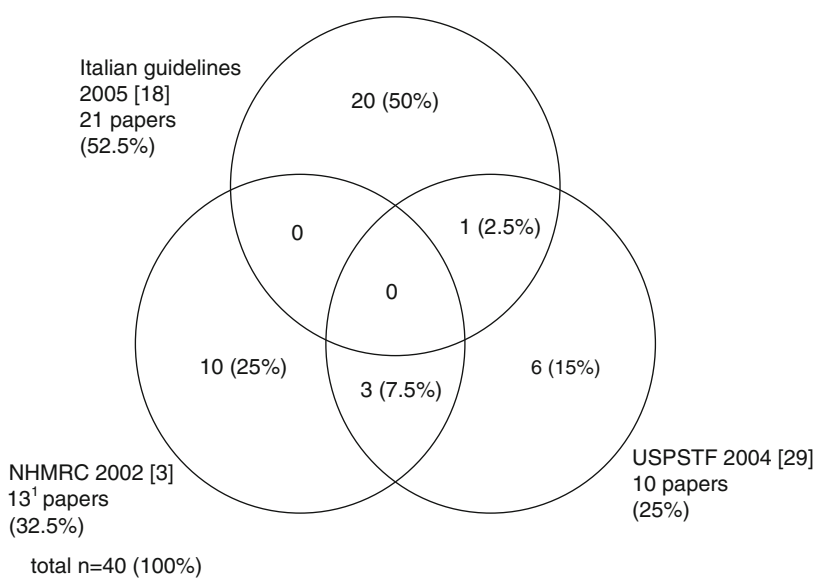

Fig. 4 Venn diagram illustrating the overlap of older reviews included in current recommendations from NHMRC, USPSTF, and Italian guidelines. Numbers and percentages inside the circles show the overlap of studies included in the four reviews. Percentages outside the circles illustrate the percentage of all 40 studies included in each of the three reviews. Total $n=40(100 \%)$. NHMRC National Health and Medical Research Council, Australia; USPSTF U.S. Preventive Services Task Force; Italian guidelines-"Italian guidelines on rehabilitation treatment of adolescents with scoliosis or other spinal deformities"; 1 quality assessment of four articles is documented in the report, the remaining papers are described narratively 


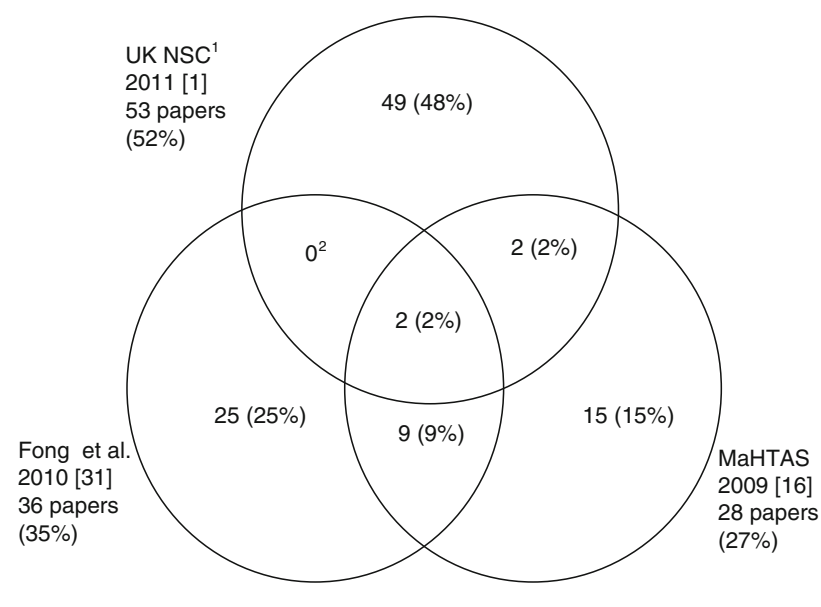

total $n=102(100 \%)$

Fig. 5 Venn diagram illustrating the overlap of papers included in the more recent systematic reviews. Numbers and percentages inside the circles show the overlap of studies included in the three reviews. Percentages outside the circles illustrate the percentage of all 40 studies included in each of the three reviews. Total $n=102(100 \%)$. UK NSC the UK National Screening Committee; MaHTAS Health Technology Assessment Section, Ministry of Health Malaysia; 1 this review also included the systematic review by Fong et al.; 2 UK NSC review cites Fong et al.

The Italian review covering a similar time period is based on 21 papers, of which only one overlaps with the USPSTF and none with the NHMRC recommendations, respectively (Fig. 4).

Further, the 2011 UK NSC review, referring to 53 publications, overlaps with only two papers in the MaHTAS review but does not overlap with any papers included in the meta-analysis by Fong et al. (although this paper is cited). In brief the three papers, developed within similar time frames, only have 2 or $2 \%$ of included studies that overlap (Fig. 5).

Table A3 (supplementary material) shows how the studies included in the analyzed reviews were contrasted in order to produce the Venn diagrams.

\section{Discussion}

Brief summary of results

\section{Searching process and selection of the reviews}

Comprehensive literature searches and selection of retrieved papers (Box 2, Fig. 2, Table A2-supplementary material) resulted in including six systematic reviews $[1,3$, $16,18,30,32]$, of which five $[1,3,16,18,30]$ were conducted in the process of systematically developing recommendations. Full text analyses of a further 34 papers resulted in excluding 15 reviews addressing the subject matter, but not fulfilling the minimal criteria for a systematic review. Nineteen full texts were also excluded for other different reasons (Fig. 1, Table A2-supplementary material). Therefore, to our knowledge this study presents the first comprehensive and in-depth analysis of systematic reviews regarding screening programs for adolescents for idiopathic scoliosis.

\section{Quality and recency of the reviews}

Overall, the quality of systematic reviews ranged from the comparatively recent (2009) moderate quality (AMSTAR score 6) Fong et al. [32] and Sabirin et al. [16] through to the outdated (2002) moderate quality NHMRC, reviews [3], to the poor quality recent (2011) UK NSC [1] and outdated (2004, nonetheless used for recent (2012) recommendations $[24,25]$ ) USPSTF [30] and Italian (2005) [18], reviews.

The reviews which supported the recommendation for school screening $[16,18,32]$ as well as those recommending against screening $[1,3,30]$ are based on different papers selected for inclusion (Figs. 2, 3, 4, 5) [and thus on different evidence or assumptions of the absence of evidence (Table 2)]. Further, regardless of the different time limits used within searches, the reviews also differed significantly with regard to the databases selected, and other resources, searched (Table 2). Moreover, significant heterogeneity was found within the reviews as follows: different research designs were considered (prospective trials and retrospective observational studies, systematic reviews, editorials), which were, except in the Fong et al. [32] meta-analysis, analyzed separately (Table 2). Conclusions were based on different criteria as follows: the set of criteria for appraising - feasibility, effectiveness and appropriateness of a screening program [1, 3], accuracy of screening tests [16, 18, 32], treatment effectiveness as a criterion justifying the need for screening $[16,18,30]$ and cost-effectiveness $[1,16]$.

\section{Methodology issues}

The reviews included within this study were heterogeneous, both with regard to the research questions asked as well as the methodology used for their development. Therefore, we conducted an umbrella review, as described by Grant and Booth [52] ${ }^{1}$ and also by Ioannidis [53]. No

\footnotetext{
${ }^{1}$ This is a review which "focuses on a broad condition or problem for which there are competing interventions and highlights reviews that address these interventions and their results" identifies component reviews, but not primary studies, provides quality assessment of studies within component reviews and/or of reviews themselves component reviews, with graphical and tabular synthesis with narrative commentary, and analysis of what is known, what remains unknown, and with recommendations for practice and future research, [52].
} 


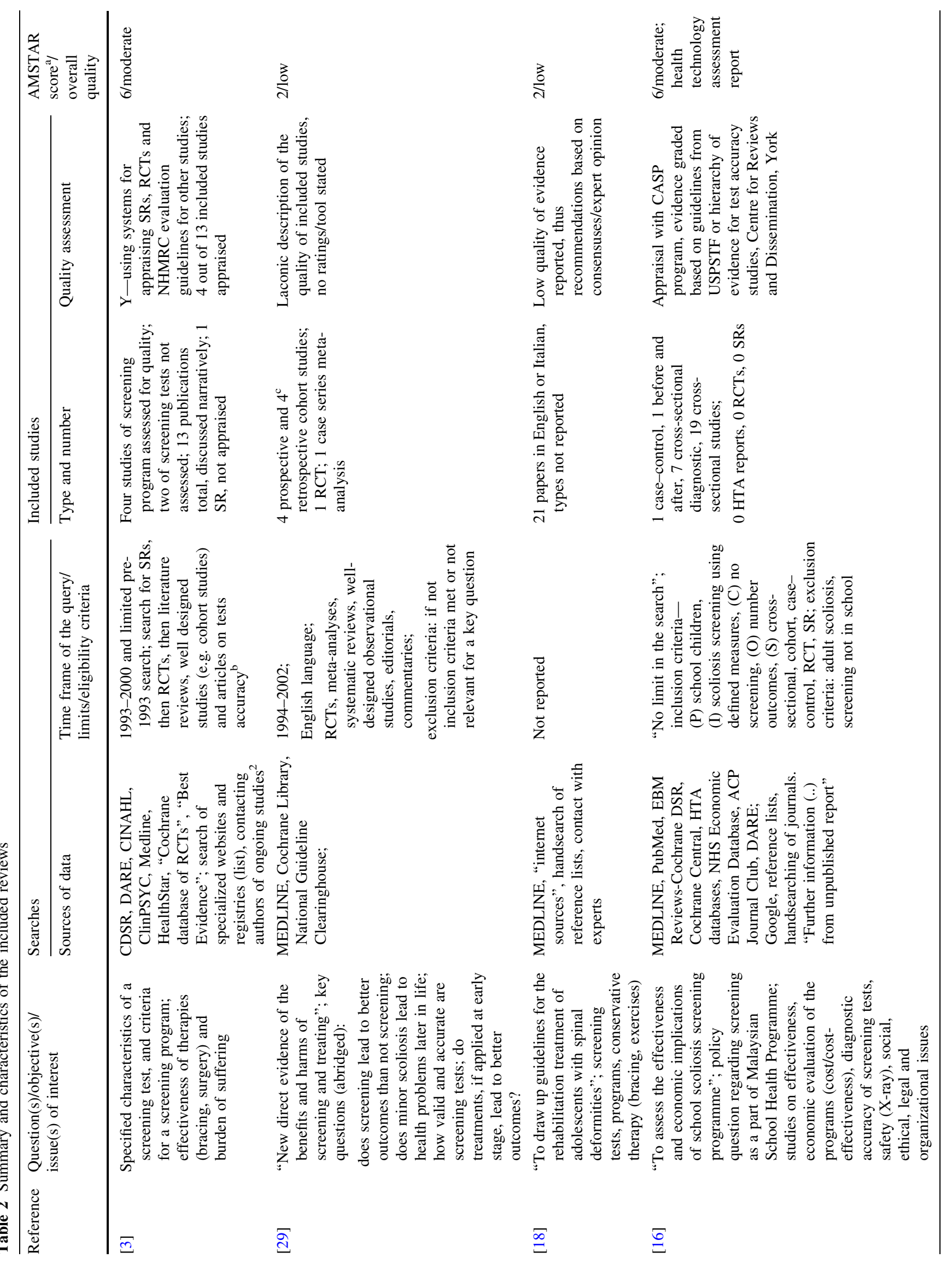




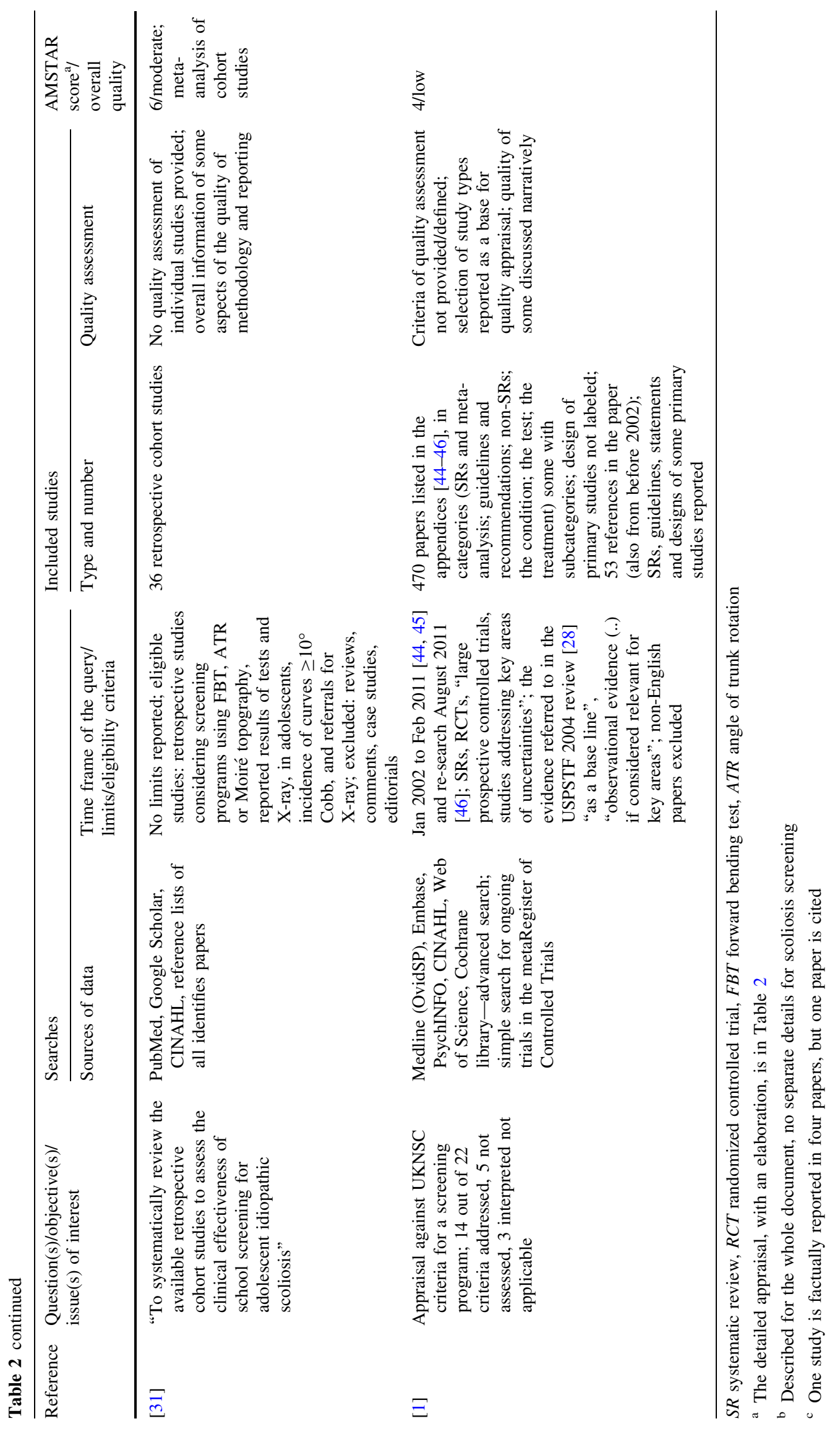


primary studies were considered. It is important to note that the term "umbrella review" is also used by some authors [e.g. 54] to describe a systematic review of high-quality systematic reviews which is not the case in this evaluation.

Discussion and critique of included reviews

\section{Higher quality systematic reviews}

Three of the systematic reviews were found to be of moderate quality; Fong et al. [10], Sabirin et al. [16] and NHMRC [3].

Fong et al. [32] (2010) reported that only $17 \%$ of the primary studies included within their meta-analysis of retrospective cohort studies found screening to be ineffective. The authors advocate for school screening, but recommended that the forward bend test should not be used alone within screening programs and that large, retrospective cohort studies are needed to better study the reliability of screening tests. As regards the studies included for analysis, the results of the Fong review only overlaps partially with the Sabirin et al. [16] study, but shows no overlap with the UK NSC 2011 review [1] (Fig. 5) and the older reviews reported by the USPSTF [30] (Fig. 2) and NHMRC [3] (Fig. 3). The conclusions from the Fong study were in part based on different evidence as well as different assumptions to the other reviews. Whilst the AMSTAR score for this meta-analysis is only of moderate quality (6), the report does meet the essential criteria of a good quality quantitative systematic review (Tables 2 and 3).

MAHTaS (2009) systematic review by Sabirin et al. [16] included a methodological assessment of individual studies as well as a cost-utility analysis. The authors recommended continuation of the screening program in Malaysia, under specific conditions: in high-risk group of 12-year-old girls, using combination of screening tests, and with wellsecured training, manpower, good referral system, treatment and funding. The review was clearly reported (AMSTAR score of 6 (Table 3); however, we encountered difficulties with regard to the assessment of question 8 within AMSTAR: unfortunately, only the results of 8 out of 24 papers appraised, utilizing the CASP tool were reported. Furthermore, studies of different types designs were included and analyzed separately (Table 2), which in some instances may diminish the internal validity of the review as well as the strength of the conclusions.

The NHMRC (2002) document [3] recommends against screening for scoliosis. All the criteria for conducting a systematic review are described in detail. Moreover, unlike the other reviews evaluated, a defined methodology for the quality assessment of systematic reviews was provided. Nonetheless, the meta-analysis included in the systematic review of evidence regarding screening for scoliosis [55], and 8 out of 12 included primary studies, were only discussed descriptively (Table 2 ), without conducting a critical appraisal of their quality. Moreover, several other eligible primary studies were not included for analysis (Fig. 4).

The NHMRC report has not been updated since 2002, while the AMSTAR does not reflect the recency of the analyzed reviews. Also, the NHMRC review [3] was not developed according to the subsequently (2009) improved NHMRC evidence hierarchies [56].

In fact, most of the six reviews analyzed matched neither the improved (2009) NHMRC [56], nor the new (2011) Oxford Centre for Evidence-Based Medicine (OCEBM) levels of evidence hierarchy (Levels of Evidence 2 [57]), with the exception of Fong et al., which can be classified as a level 3 evidence in the OCEBP classification (Box 3).

Box 3 Definitions of evidence hierarchy for diagnostic accuracy and screening interventions described by different organizations

\section{Diagnostic and monitoring tests/diagnostic accuracy}

The new (2011) Oxford Centre for Evidence-Based Medicine (OCEBM) levels of evidence hierarchy (Levels of Evidence 2 [56] ) states that "systematic review of cross sectional studies with consistently applied reference standard and blinding" are step 1 (level 1) of evidence and consequently individual studies are level II evidence;

The improved (2009) Australian NHMRC “Additional Levels of Evidence and Grades for Recommendations for Developers of Guidelines" document [55], providing hierarchy of evidence for different types of research questions, defines level II evidence as "a study of test accuracy with: an independent, blinded comparison with a valid reference standard, among consecutive persons with a defined clinical presentation", and systematic reviews of level II studies are level I evidence;

Both classifications list case-control studies (level IV-OCEBM, level III-3-NHMRC) but not retrospective cohort studies.

Screening interventions (both OCEBM and NHMRC)

Systematic reviews of prospective, randomized controlled trials (RCTs)_level I evidence;

Individual RCTs-level II evidence;

\section{Lower quality reviews}

The UK NSC review (2011) [1], prepared as an update of the 2004 USPSTF analysis [30], sustains the recommendations against screening. The report scored 4 with AMSTAR (low quality review, Tables 2 and 3). The authors addressed several criteria regarding the mandate for a screening program, including the cost-effectiveness of the programs, as well as the effectiveness of conservative and surgical treatment. The observational, experimental studies as well as the systematic reviews included within this 
Table 3 AMSTAR ratings for included reviews

\begin{tabular}{|c|c|c|c|c|c|c|c|c|c|c|c|c|c|}
\hline \multirow[t]{2}{*}{ Reference } & \multicolumn{11}{|c|}{ AMSTAR questions $^{\mathrm{a}}$} & \multirow[t]{2}{*}{ Total Yes } & \multirow[t]{2}{*}{ Overall quality } \\
\hline & 1 & 2 & 3 & 4 & 5 & 6 & 7 & 8 & 9 & 10 & 11 & & \\
\hline [3] & $\mathrm{Y}$ & CA & $\mathrm{Y}$ & $\mathrm{Y}$ & $\mathrm{N}$ & $\mathrm{Y}$ & $\mathrm{Y}$ & $\mathrm{N}$ & NA & $\mathrm{N}$ & $\mathrm{N}$ & 5 & Moderate \\
\hline$[29]^{\mathrm{c}}$ & $\mathrm{Y}$ & CA & $\mathrm{N}^{\mathrm{d}}$ & $\mathrm{N}$ & $\mathrm{N}$ & $\mathrm{Y}$ & $\mathrm{N}$ & $\mathrm{N}$ & NA & $\mathrm{N}$ & $\mathrm{N}$ & 2 & Low \\
\hline [18] & $\mathrm{Y}$ & $\mathrm{CA}$ & $\mathrm{N}^{\mathrm{e}}$ & $\mathrm{Y}$ & $\mathrm{N}$ & $\mathrm{N}$ & $\mathrm{N}^{\mathrm{f}}$ & $\mathrm{N}^{\mathrm{g}}$ & NA & $\mathrm{N}$ & $\mathrm{N}$ & 2 & Low \\
\hline [16] & $\mathrm{Y}$ & $\mathrm{Y}$ & $\mathrm{Y}$ & $\mathrm{Y}$ & $\mathrm{Y}$ & $\mathrm{Y}$ & $\mathrm{CA}^{\mathrm{h}}$ & $\mathrm{N}^{\mathrm{i}}$ & $\mathrm{NA}^{\mathrm{j}}$ & $\mathrm{N}$ & $\mathrm{N}$ & 6 & Moderate \\
\hline [31] & $\mathrm{Y}$ & $\mathrm{Y}$ & $\mathrm{Y}$ & $\mathrm{N}$ & $\mathrm{N}$ & $\mathrm{Y}$ & $\mathrm{N}^{\mathrm{k}}$ & $\mathrm{N}^{\mathrm{g}}$ & $\mathrm{Y}$ & $\mathrm{Y}^{1}$ & $\mathrm{~N}$ & 6 & Moderate \\
\hline [1] & $\mathrm{Y}$ & $\mathrm{CA}$ & $\mathrm{Y}$ & $\mathrm{Y}$ & $\mathrm{N}$ & $\mathrm{Y}$ & $\mathrm{N}$ & $\mathrm{N}^{\mathrm{g}}$ & NA & $\mathrm{N}$ & $\mathrm{N}$ & 4 & Low \\
\hline
\end{tabular}

$Y$ yes, $N$ no, $C A$ cannot answer, $N A$ not applicable

${ }^{a}$ Questions [38]: “1 Was an a priori design provided?, 2 Was there duplicate study selection and data extraction?, 3 Was a comprehensive literature search performed?, 4 Was the status of publication (i.e. grey literature) used as an inclusion criterion?, 5 Was a list of studies (included and excluded) provided? 6 Were the characteristics of the included studies provided?, 7 Was the scientific quality of the included studies assessed and documented?, 8 Was the scientific quality of the included studies used appropriately in formulating conclusions?, 9 Were the methods used to combine the findings of studies appropriate?, 10 Was the likelihood of publication bias assessed?, 11 Were potential conflicts of interest included?"

${ }^{\mathrm{b}}$ Scores of 0-4 indicate that the review is of low quality, 5-8 of moderate quality and 9-11 of high quality [41]

c As the 2004 USPSTF statement, and the subsequent Guides, were based on, and referred to, the 2004 "Brief Evidence Update", we focused our appraisal on that document, and referred to other related documents only if indicated/referenced to in the "Brief Evidence Update": (1) [27], pp. xlvii-liv, 517-529, (2) [26, 42]; (3) [43]

d The 1996 document [27] provides sufficient information for a Y, but the 2004 update reports only on databases searched, with no information on supplementary strategies

e Only one electronic source reported; the phrase "internet sources" does not fulfil the criteria of AMSTAR scoring

${ }^{\mathrm{f}}$ A hierarchy of study quality was used, and the authors report on low quality of analyzed papers, but no quality assessment is documented

g Rated "N" in accordance with AMSTAR criteria, because item 7 rated "N"

${ }^{\text {h }}$ Quality of 8 (7 cross-sectional and 1 case-control), out of 28 included studies, were assessed with CASP tool, but remaining 16 cross-sectional, 2 cost-effectiveness, 1 before-and-after study, and 1 study described as cross-sectional/case series, were not; also, answers to the CASP questions are reported without an elaboration; therefore, according to the instructions for AMSTAR [38], we rated this item with "CA"

i A discussion regarding the quality of included studies is provided, but we rated "N", because item 7 rated "N"

$\mathrm{j}$ The protocol of the review includes information about planned analysis for heterogeneity, but no information is provided in the actual report

${ }^{\mathrm{k}}$ The authors did discuss the scientific quality of the included studies, but they did not use any quality scoring tool or a checklist separately for each of the included studies, thus we rated "N"

${ }^{1}$ Funnel plots are discussed in the article and included in the thesis [48], which we excluded as a duplicate; the remaining contents of these two papers are identical

paper discussed diverse aspects of scoliosis management and are described broadly and narratively. Eligibility criteria for paper inclusion are not reported. The lists of 470 retrieved reports [45-47] supplement the review, which factually discusses 53 papers but no list of excluded studies is provided. The review reports on search limits from 2002 to 2011, but single older studies are also included. Thus, it would be difficult to judge how the studies described in the review were chosen. Further, while the hierarchy of evidence level is suggested - the designs of the discussed papers are reported, crucially the included studies are not critically appraised. Without knowledge of the methodological quality of included papers it is very difficult to come to a conclusion as to whether the results of this review can be applied to practice and whether the recommendations are truly valid and evidence-based.
The USPSTF resource review for the 2012 USPSTF recommendations against screening [24, 25] - the 2004 "Brief Evidence Update" [30], recommending against school screening - has been shown to be of poor quality, with an AMSTAR score of 2. The "critical" "key question"- "Is there new evidence that scoliosis treatment leads to better health outcomes if applied at an early stage", was answered by analyzing one RCT, three reports of a large retrospective observational studies, one case series and a meta-analysis of studies from between 1975 and 1995 on the effectiveness of conservative treatments [55]. No list of excluded studies was provided, and crucially no methodological quality appraisal of their included studies was conducted. Further, the search period is outdated (1994-2002) and the literature search was not comprehensive (Fig. 4). Consequently, this review scored a low value of 2 with AMSTAR (Table 3). Whilst the USPSTF did 
elaborate on the development process for their recommendations, including the process of the evidence review [27, $28,43,44]$, this development process does not seem to have been followed in their study (Tables 2 and 3). Nonetheless, when appraising the review, we considered the USPSTF methodology descriptions current for that period of time, described elsewhere [27, 28, 43, 44] (Table 3). Otherwise, the review would have attained an even lower score. As the review was published in 2004, the present USPSTF methodology of guideline development, improved subsequently in 2007 and 2008 [58-60], was not analyzed. It also means that the current (2012) USPSTF recommendations against scoliosis screening are based on the outdated methodology of the USPSTF guideline development.

Finally, in the USPSTF "review of the process" paper the authors say that comprehensive systematic reviews are "long, detailed reports of interest to a minority of readers and of limited value to busy clinicians" [44]. It is difficult to agree with this notion, as researchers, clinicians, commissioners and service users routinely make use of conclusions/recommendations from systematic reviews (e.g. Cochrane reviews with separately published summaries). In fact, systematic reviews are considered to be equivalent to research studies that produce the highest level of evidence according not only to OCEBM [57] and NHMRC [56] but to numerous other organizations. Additionally, the detailed guidelines for reporting explicit systematic reviews of different types of studies have been in use (such as QUORUM (1999) [61], then PRISMA (2009) [38], MOOSE (2000) [62], and reporting guidelines from the US Institute of Medicine (2011) [63]).

The authors of the Italian guidelines (2005) [18], recommending for school screening, reported on a systematic analysis of the literature. It achieved an AMSTAR score of 2 (Table 3). A classification of the levels of evidence is provided in this paper, including expert opinions. Within these recommendations the evidence for scoliosis screening was classified as "E2: fair scientific consensus" [18]. In brief, the document provides a recommendation on screening for scoliosis driven from a consensus of a panel of experts, rather than on a systematic analysis of available evidence.

Strengths and limitations of the paper

The database search was supplemented with an inspection of dedicated registries as well as websites of organizations (e.g. International Network of the Agencies of Health Technology Assessment). This resulted in retrieving important publications (UK NSC [1], NHMRC [3] and MaHTAS [16]) not indexed in major databases (as reported in detail in Fig. 3) and omitted in recent reviews [4, 64, 65]. Reference lists of publications that were retrieved were also hand searched. Potential authors of unpublished data were not contacted, as the aim of this study was to appraise published systematic reviews rather than performing a meta-analysis of primary studies. The findings from the grey literature search revealed that another systematic review was currently in press [51]. Another limitation to this paper was the fact that the papers included within this review were not fully congruent with regard to the research objectives, questions posed, strategies of review development and reporting. We believe that selection bias was unlikely in this study as only reviews meeting the specified inclusion criteria were selected.

Finally, as to our knowledge this is the first evidencebased appraisal of systematic reviews addressing school screening for scoliosis, the findings of this review could not be compared to similar reports.

\section{Conclusions}

The results found within this paper appear on the surface to be very controversial. However, further detailed inquiry based on in-depth evaluations of the methodological quality of included papers within this reviews provide much needed clarification. The most recent and current reviews provided by Fong et al. in 2010 and Sabirin et al. in 2009 both of which scored a moderate quality 6 made recommendations to continue school screening programs. These reviews were based on papers that were current, and most crucially were evaluated for their methodological quality so that readers can be assured of the reliability and validity of their results and conclusions.

The results presented within our evaluation suggest that many of the recommendations made against school screening are based on research papers that are over 10 years old and are, therefore, outdated. More to the point and of utmost importance, most of these recommendations did not evaluate the methodological quality of the papers they included within their reviews. Thus it is impossible to know whether these results and consequently their recommendations are truly valid and can reliably and justifiably be applied to clinical practice. Until such time, therefore, we suggest that the recommendations based on low-quality reviews need to be interpreted with caution.

Implications for research and practice

There is an urgent need for high-quality primary studies to be conducted on school screening for scoliosis to provide a definitive answer to this very important question. Although very difficult to conduct and not unanimously recommended in cases of school screening programs (Box 3, Fong et al. [32]), RCTs are indicated in the latest 
classifications of the Hierarchy of Evidence as the second highest level of evidence from primary diagnostic studies which investigate important patient outcomes [66]. The first or highest level of evidence is systematic reviews of such papers. In practice, however, we acknowledge that an RCT comparing a group undergoing school screening to one not undergoing screening would be very difficult if not impossible to conduct. Whilst Fong recommended retrospective studies, the Cochrane Back Review Group does not currently recognize such research designs to be of sufficiently good quality to be included within a Cochrane back review due to the high degree of error resulting from bias and confounding variables [67]. What we would recommend, however, are PROSPECTIVE cohort studies with a control group, with participants matched for age, gender and other key variables.

In brief, once a number of prospective studies with a control group have been conducted then well-conducted systematic reviews which are crucially needed can be carried out. Standards similar to those found within the Cochrane collaboration and other similar organizations would need to be followed to provide the much needed reliable and trustworthy evidence for researchers, clinicians, commissioners and most importantly for the patients and families concerned.

\section{Protocol registration}

This paper reports on a section of an overview of systematic reviews regarding nonsurgical management of idiopathic scoliosis, including screening and is registered at PROSPERO, CRD York, CRD42013003538.

\section{Conflict of interest None.}

Open Access This article is distributed under the terms of the Creative Commons Attribution License which permits any use, distribution, and reproduction in any medium, provided the original author(s) and the source are credited.

\section{References}

1. [No authors listed] (2009) Screening for adolescent scoliosis. External review against programme appraisal criteria for the UK National Screening Committee (UK NSC). Bazian Ltd. http:// www.screening.nhs.uk/scoliosis. Accessed 11 June 2013

2. Labelle H, Richards SB, De Kleuver M, Grivas TB, Luk KD, Wong HK, Thometz J, Beauséjour M, Turgeon J, Fong DY (2013) Screening for adolescent idiopathic scoliosis: an information statement by the scoliosis research society international task force. Scoliosis 8:17

3. National Health and Medical Research Council (2002) Child health screening and surveillance: a critical review of the evidence. Centre for Community Child Health, Royal Children's Hospital Melbourne

4. Linker BA (2012) A dangerous curve. The role of history in America's scoliosis screening programs. Am J Public Health 102:1106-1116

5. Taylor TK, Bushell G, Ghosh P (1978) School screening for scoliosis: a Pandora's box. Aust N Z J Surg 48:2-3

6. Burwell G (1988) The British decision and subsequent events. Spine 13:1192-1194

7. Winter RB, Lonstein JB (1997) To brace or not to brace: the true value of school screening. Spine 22:1283-1284

8. Dickson RA, Weinstein SL (1999) Bracing (and screening)-yes or no? J Bone Jt Surg Br 81:193-198

9. Winter RB, Banta JV, Engler G, Scoliosis Research Society (1995) Screening for scoliosis. JAMA 273:185-186

10. Sox HC Jr, Woolf SH, US Preventive Services Task Force (1995) In reply (Screening for scoliosis). JAMA 273:186

11. Bunnell WP (2005) Selective screening for scoliosis. Clin Orthop Relat Res 434:40-45

12. Bunge EM, de Koning HJ (2006) Selective screening for scoliosis. Clin Orthop Relat Res 445:277-278

13. Bunge EM, Juttmann RE, van Biezen FC, Creemers H, Hazebroek-Kampschreur AAJM, Luttmer BCF, Wiegersma A, de Koning $\mathrm{H}$, for the Netherlands Evaluation Study for Screening for Scoliosis (NESCIO) group (2008) Estimating the effectiveness of screening for scoliosis: a case-control study. Pediatrics 121:9-14

14. Richards BS, Beaty JH, Thompson GH, Willis RB (2008) Estimating the effectiveness of screening for scoliosis. Pediatrics 121:1296-1297

15. School Health Connections/Healthy Start Office (2007) The Standards for Scoliosis Screening in California Public Schools. California Department of Education

16. Sabirin J, Buang SN (2009) School scoliosis screening programme. Health Technology Assessment Report, Health Technology Assessment Section, Ministry of Health Malaysia, MOH/ P/PAK/186.09 (TR). www.moh.gov.my/health_assessments/58

17. Richards BS, Vitale M Screening for Idiopathic Scoliosis in Adolescents. AAOS-SRS-POSNA-AAP Position Statement. http://www.aaos.org/about/papers/position/1122.asp. Accessed 13 June 2013

18. Negrini S, Aulisa L, Ferraro C, Fraschini P, Masiero S, Simonazzi P, Tedeschi C, Venturin A (2005) Italian guidelines on rehabilitation treatment of adolescents with scoliosis or other spinal deformities. Eura Medicophys 41:183-201

19. Grivas TB, Wade MH, Negrini S, O'Brien JP, Maruyama T, Hawes MC, et al (2007) SOSORT consensus paper: school screening for scoliosis. Where are we today? Scoliosis 2:17 http:// www.scoliosisjournal.com/content/2/1/17

20. Negrini S, Aulisa AG, Aulisa L, Circo AB, de Mauroy JC, Durmala J, et al (2012) 2011 SOSORT guidelines: Orthopaedic and rehabilitation treatment of idiopathic scoliosis during growth. Scoliosis 7:3 http://www.scoliosisjournal.com/content/7/1/3

21. UK Screening Portal, UK National Screening Committee, UK National Screening Policy Database. Scoliosis. http://www. screening.nhs.uk/policydb.php./; http://www.screening.nhs.uk/ scoliosis. Accessed 15 June 2013

22. US Preventive Services Task Force (2004) Screening for idiopathic scoliosis in adolescents: recommendation statement. http:// www.ahrq.gov. Accessed 15 Jan 2013

23. American Academy of Family Physicians (2012) Summary of recommendations for clinical preventive services. American Academy of Family Physicians (AAFP), Leawood, p 18

24. Agency for Healthcare Research and Quality (2012) Guide to Clinical Preventive Services. AHRQ Publication No. 12-05154, Rockville, p. 71. www.ahrq.gov/clinic/pocketgd.htm. Accessed 20 Feb 2013 
25. US Preventive Services Task Force (2012) Recommendations. http://www.uspreventiveservicestaskforce.org/recommendations. htm. Accessed 23 Feb 2013

26. Canadian Task Force on Periodic Health Examination (1979) The periodic health examination. CMA J 121:1193-1254

27. US Preventive Services Task Force (1993) Screening for adolescent idiopathic scoliosis: policy statement. JAMA 269:2664-2666

28. US Preventive Services Task Force (1996) Guide to Clinical Preventive Services: Report of the U.S. Preventive Services Task Force. 2nd edn. US Department of Health and Human Services, Washington (DC). www.ncbi.nlm.nih.gov/books/NBK15435/

29. Goldbloom R (1994) Screening for Adolescent Idiopathic Scoliosis. In: Canadian Task Force on Periodic Health Examination. The Canadian guide to clinical preventive health care. Minister of Public Works and Government Services Canada, Ottawa. http:// canadiantaskforce.ca/wp-content/uploads/2013/03/Chapter31_ idio_adoles_scoliosis94.pdf?9d7bd4

30. [No authors listed] (2004) Screening for idiopathic scoliosis in adolescents: a brief evidence update for the U.S. preventive services task force. AHRQ Pub. No. 05-0568-B http://www.uspre ventiveservicestaskforce.org/3rduspstf/scoliosis/scolioup.pdf

31. Richards BS, Vitale MG (2008) Screening for idiopathic scoliosis in adolescents. An information statement. J Bone Jt Surg Am 90:195-198

32. Fong DY, Lee CF, Cheung KM, Cheng JC, Ng BK, Lam TP, Mak KH, Yip PS, Luk KD (2010) A meta-analysis of the clinical effectiveness of school scoliosis screening. Spine 35:1061-1071

33. Larson N (2011) Early onset scoliosis: what the primary care provider needs to know and implications for practice. J Am Acad Nurse Pract 23:392-403

34. Wilkinson J, Bass C, Diem S, Gravley A, et al (2012) Institute for Clinical Systems Improvement. Preventive Services for Children and Adolescents. Bloomington (MN): Institute for Clinical Systems Improvement (ICSI); p 87 http://bit.ly.PrevServKids0912

35. Sabirin J, Bakri R, Buang SN, Abdullah AT, Shapie A (2010) School scoliosis screening programme-a systematic review. Med J Malaysia 65:261-267

36. Petticrew M (2003) Why certain systematic reviews reach uncertain conclusions. BMJ 326:756-758

37. Smith V, Devane D, Begley CM, Clarke M (2011) Methodology in conducting a systematic review of systematic reviews of healthcare interventions. BMC Med Res Methodol 11:15

38. Moher D, Liberati A, Tetzlaff J, Altman DG (2009) Preferred reporting items for systematic reviews and meta-analyses: the PRISMA statement. BMJ 339:b2535

39. [No authors listed] (2005) Glossary of terms in the cochrane Collaboration. version 4.2.5., The cochrane collaboration

40. Connor Gorber S, Singh H, Pottie K, Jaramillo A, Tonelli M (2012) Process for guideline development by the reconstituted Canadian Task Force on Preventive Health Care. CMAJ 184:1575-1581

41. Shea BJ, Grimshaw JM, Wells GA, Boers M, Andersson N, Hamel C, Porter AC, Tugwell P, Moher D, Bouter LM (2007) Development of AMSTAR: a measurement tool to assess the methodological quality of systematic reviews. BMC Med Res Methodol 7:10

42. Popovic I, Windsor B, Jordan V, Showell M, Shea B, Farquhar CM (2012) Methodological quality of systematic reviews in subfertility: a comparison of two different approaches. PLoS One 7(12):e50403

43. Sox HC Jr, Berwick DM, Berg AO et al (1993) Screening for adolescent idiopathic scoliosis: review article. JAMA 269:2667-2672

44. Harris RP, Helfand M, Woolf SH, Lohr KN, Mulrow CD, Teutsch SM, Atkins D (2001) Current methods of the US Preventive Services Task Force: a review of the process. Am J Prev Med 20(3 Suppl):21-35
45. [No authors listed] (2011) Knowledge update on screening for scoliosis (February 2011). Available at http://www.screening.nhs. uk/scoliosis. Accessed 11 June 2013

46. [No authors listed] (2011) Appendix to knowledge update on screening for scoliosis. http://www.screening.nhs.uk/scoliosis. Accessed 11 June 2013

47. [No authors listed] (2011) August 2011 update to the February 2011 Knowledge update on screening for scoliosis. http://www. screening.nhs.uk/scoliosis. Accessed 11 June 2013

48. [No authors listed] (2002) Child Health Screening and Surveillance: Supplementary Document-Context And Next Steps. The Child and Youth Health Inter-governmental Partnership, National Public Health Partnership. http://www.nphp.gov.au. Accessed 31 May 2013

49. Lee CF (2009) School screening and curve progression in adolescent idiopathic scoliosis. Dissertation, University of Hong Kong

50. Beauséjour M, Goulet L, Parent S, Feldman DE, Turgeon I, RoyBeaudry M, Sosa JF, Labelle H; Members of the Quebec Scoliosis Society and of the Canadian Paediatric Spinal Deformities Study Group (2013) The effectiveness of scoliosis screening programs: methods for systematic review and expert panel recommendations formulation. Scoliosis 8:12

51. Feldman D, Beauséjour M, Sosa JF, Goulet L, Parent S, Labelle $\mathrm{H}$; and the members of the Quebec Scoliosis Society and the Canadian Pediatric Spinal Deformities Study Group (2014) Cost effectiveness of school screening for scoliosis: a systematic review. Int J Child Adolesc Health 7(1). Available at https:// www.novapublishers.com/catalog/product_info.php?products_ id $=46272$. Accessed 19 Apr 2014

52. Grant MJ, Booth A (2009) A typology of reviews: an analysis of 14 review types and associates methodologies. HILJ 26:91-108

53. Ioannidis JPA (2009) Integration of evidence from multiple metaanalyses: a primer on umbrella reviews, treatment networks and multiple treatments meta-analyses. CMAJ 181:488-493

54. Moe RH, Haavardsholm EA, Christie A, Jamtvedt G, Dahm KT, Hagen KB (2007) Effectiveness of nonpharmacological and nonsurgical interventions for hip osteoarthritis: an umbrella review of high quality systematic reviews. Phys Ther $87: 1716-1727$

55. Rowe DE, Bernstein SM, Riddick MF, Adler F, Emans JB, Gardner-Bonneau D (1997) A meta-analysis of the efficacy of non-operative treatments for idiopathic scoliosis. J Bone Jt Surg Am 79:664-674

56. Centre for Evidence-Based Medicine, Oxford, UK (2011) Levels of evidence for primary research question. www.cebm.net. Accessed 15 May 2013

57. National Health and Medical Research Council (2009) NHMRC Levels of evidence and grades of recommendations for developers of guidelines. http://www.nhmrc.gov.au/guidelines/resour ces-guideline-developers. Accessed 30 May 2013

58. Guirguis-Blake J, Calonge N, Miller T, Siu A, Teutsch S, Whitlock E, U.S. Preventive Services Task Force (2007) Current processes of the U.S. Preventive Services Task Force: refining evidence-based recommendation development. Ann Intern Med 147:117-122

59. Barton MB, Miller T, Wolff T, Petitti D, LeFevre M, Sawaya G, Yawn B, Guirguis-Blake J, Calonge N, Harris R, U.S. Preventive Services Task Force (2007) How to read the new recommendation statement: methods update from the U.S. Preventive Services Task Force. Ann Intern Med 147(2):123-127

60. U.S. Preventive Services Task Force Procedure Manual (2008) AHRQ Publication No. 08-05118-EF. http://www.uspreventive servicestaskforce.org/uspstf08/methods/procmanual.htm

61. Moher D, Cook D, Eastwood S, Olkin I, Rennie D, Stroup DF, for the QUORUM Group (2009) Improving the quality of reports of 
meta-analyses of randomised controlled trials: the QUORUM statement. Lancet 354:1896-1900

62. Stroup DF, Berlin JA, Morton SC, Olkin I, Williamson GD, Rennie D, Moher D, Becker BJ, Sipe TA, Thacker SB, for the Meta-analysis of Observational Studies in Epidemiology (MOOSE) Group (2000) Meta-analysis of observational studies in epidemiology. A proposal for reporting. JAMA 283:2008-2012

63. IOM (Institute of Medicine) (2011) Standards for reporting systematic reviews. In: Finding What Works in Health Care: Standards for Systematic Reviews. The National Academies Press, Washington DC, Chapter 5

64. Hresco MT (2013) Idiopathic scoliosis in adolescents. N Engl J Med 368:834-841
65. Altaf F, Gibson A, Dannawi Z, Noordeen H (2013) Adolescent idiopathic scoliosis. BMJ 2013(346):f2508

66. The Joanna Briggs Institute Levels of Evidence and Grades of Recommendation Working Party (2014) Supporting Document for the Joanna Briggs Institute Levels of Evidence and Grades of Recommendation. The Joanna Briggs Institute. http://joannab riggs.org/assets/docs/approach/Levels-of-Evidence-Supporting Documents.pdf. Accessed 15 March 2014

67. Bettany-Saltikov J, Weiss HR, Chockalingam N, Taranu R, Srinivas S, Hogg J, Whittaker V (2013) Kalyan RV (2013) Surgical versus non-surgical interventions in patients with adolescent idiopathic scoliosis (Protocol). Cochrane Database of Systematic Reviews 7:CD010663 